\title{
Room-Temperature Terahertz Detection and Imaging by Using Strained-Silicon MODFETs
}

\author{
Juan Antonio Delgado-Notario, Vito Clericò, \\ Kristel Fobelets, Jesús Enrique Velázquez-Pérez and \\ Yahya Moubarak Meziani
}

Additional information is available at the end of the chapter

http://dx.doi.org/10.5772/intechopen.76290

\begin{abstract}
This chapter reports on an experimental and theoretical study of Schottky-gated strainedSi modulation-doped field-effect transistors (MODFETs) with different sub-micron gate lengths $(100,250$, and $500 \mathrm{~nm})$. Room-temperature detection of terahertz $(\mathrm{THz})$ radiation by the strained-Si MODFETs was performed at two frequencies $(0.15$ and $0.3 \mathrm{THz})$. A technology computer-aided design (TCAD) analysis based on a two-dimensional hydrodynamic model (HDM) was used to investigate the transistor response to $\mathrm{THz}$ radiation excitation. TCAD simulation was validated through comparison with $\mathrm{DC}$ and low-frequency AC measurements. It was found that the photoresponse of the transistors can be improved by applying a constant drain-to-source bias. This enhancement was observed both theoretically and experimentally. The HDM model satisfactorily describes the experimental dependence of the photoresponse on the excitation frequency, the gate bias, and the drain-to-source current bias. The coupling of the incoming $\mathrm{THz}$ radiation to the MODFETs was studied at 0.15 and $0.3 \mathrm{THz}$. Finally, to demonstrate the suitability of strained-Si MODFET for terahertz applications, an image sensor within a pixel-by-pixel terahertz imaging system for the inspection of hidden objects was used.
\end{abstract}

Keywords: strained silicon, MODFETs, THz, plasma waves, TCAD, imaging, detectors

\section{Introduction}

The demand to substantially improve electron mobility in Si-based field effect transistors (FETs) led to the development of $\mathrm{Si} / \mathrm{Si}_{1-x} \mathrm{Ge}_{x}$ MODFETs (modulation-doped field-effect transistor). 
A MODFET is based on a single or a double heterojunction of two semiconductors with different bandgaps. In an n-channel MODFET, electrons diffuse from a highly doped layer toward a lowly doped layer of a high-mobility material where they are confined by means of the conduction-band discontinuity between the two materials. These electrons form a two-dimensional electron gas (2DEG) conductive channel at the heterointerface. Single-device strained $\mathrm{Si} / \mathrm{Si}_{0.6} \mathrm{Ge}_{0.4}$ MODFETs with 100-nm T-gates with up to 74-GHz current gain cutoff frequency and 107-GHz maximum oscillation frequency were demonstrated [1].

The terahertz (THz) region ( 0.1-10 THz; 0.03-3 mm; 3-300 $\left.\mathrm{cm}^{-1}\right)$ lies in the gap between the microwaves and infrared regions of the electromagnetic (EM) spectrum. THz radiations have extraordinary properties; it is a non-ionizing radiation and it is capable of penetrating through many non-conductive materials [2]. THz radiations have shown a great potential in a huge range of $\mathrm{THz}$ applications including astronomy [3,4], spectroscopy (rotational, vibrational, and translational modes in the $\mathrm{THz}$ range are specific to a particular substance allowing to obtain a THz fingerprint) [5-7], thickness measurement of multilayer objects [8], communications with a bandwidth significantly higher than those based on microwaves [9], nondestructive inspection based on both imaging of concealed objects and spectroscopy [10], metrology [11], quality control [12], and so on. THz rays (T-rays) permit imaging with a diffractionlimited resolution similar to that of the human eye [13], and, since common optically opaque packaging materials are transparent to T-rays, the inspection of concealed objects is possible.

During the last decade, new promising 2D materials have recently attracted interest to develop room-temperature solid-state $\mathrm{THz}$ sensors [14]. However, so far, only devices based on III-V materials [15] and silicon [16] have proved experimentally their potential to build low-cost, compact, scalable, and reliable systems; accordingly, the extension of the frequency range of these devices generates a great interest in $\mathrm{THz}$ detection.

Solid-state devices are rising as one of the most promising ways to obtain $\mathrm{THz}$ detection and emission at room temperature. Dyakonov and Shur proposed in [17-19] the use of field-effect transistors (FETs) as detectors, multipliers, and mixers in the THz range using the oscillations of the plasma waves in the channel. Nonlinear properties of the two-dimensional plasma permit the detection of the $\mathrm{THz}$ radiation. Plasma wave-based detectors can directly convert the incoming EM radiation into measurable voltage or current. They demonstrated that a FET under excitation by $\mathrm{THz}$ radiation generates a DC drain-to-source voltage when the drain is open and, therefore, operating in the photovoltaic-mode detection. The value of this voltage can be modulated by the gate-to-source bias voltage, as the gate bias controls the plasma density and therefore the carrier concentration in the FET channel. The increasing availability of continuous-wave compact sources based on solid-state oscillators in the millimeter-wave range raises the interest on the development of direct detectors [20].

This chapter presents a study of room-temperature terahertz detection using strained-silicon modulation field-effect transistors with three different gate lengths. Detection of THz radiation [21, 22] and imaging using Si/SiGe transistors have been previously demonstrated [23, 24]. A main distinct interest of the high-mobility n-type FETs based on the Si/SiGe system is that, unlike the ones based on III-V plasmon detectors, it will be easy to integrate MODFET THz detectors with mainstream Si technology circuits, since both are fabricated on conventional 
Si wafers. Therefore, strained-Si MODFETs can lead to single-chip high-performance $\mathrm{THz}$ (basic analog building blocks were already demonstrated using Si/SiGe FETs [25]) and guide to future compact, low-cost, high-speed, and high-precision $\mathrm{THz}$ detectors.

\section{Device description and TCAD simulations}

\subsection{Strained-silicon MODFET}

The devices under study are based on the Si/SiGe system, and the layout of the transistors is shown in Figure 1(a). The epistructure of the MODFETs used in this work is as follows: a thick relaxed linearly graded $\mathrm{SiGe}$ virtual substrate is grown over a p-doped conventional $\mathrm{Si}$ wafer. The final top Ge molar concentration in the virtual substrate was 0.3. The structure has an undoped 12-nm tensile strained Si channel, sandwiched between two n-doped $\mathrm{Si}_{0.70} \mathrm{Ge}_{0.30}$ relaxed supply layers (reddish layers) to generate a high density of electrons in the strainedSi quantum well $[26,27]$. The highlighted bluish layer marks out the strained-Si quantum well. Pt/Au was evaporated to fabricate the Schottky gate that was not symmetrically placed between the source and the drain. A more detailed description of the transistor fabrication can be found in [26].

The material system Si/SiGe allows the creation of a thin layer of strained silicon under tetragonal (biaxial tensile) strain due to the different values of the Si and the SiGe lattice constants. Tetragonal strain has the effect of lifting the sixfold degeneracy of the conduction band in

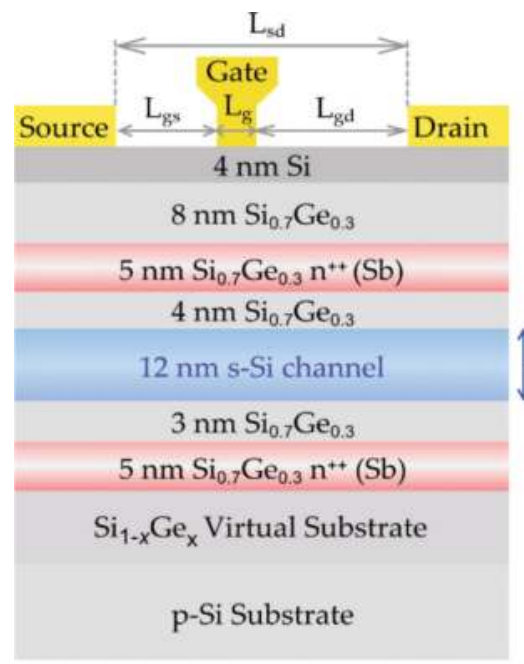

(a)

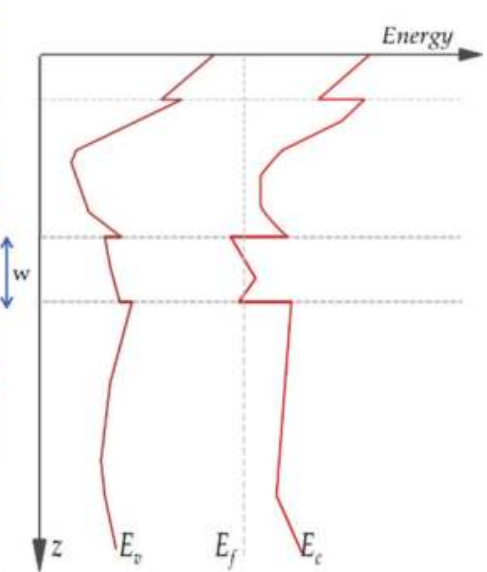

(b)

Figure 1. (a) Epistructure of the Si/SiGe MODFETs showing the vertical layout of the transistors. (b) Conduction and valence band profiles and the Fermi level under the gate in equilibrium [26]. 
silicon into a twofold and fourfold degenerate sets lowering the energy of the two valleys with their long axis perpendicular to the $\mathrm{Si} / \mathrm{SiGe}$ interface. Consequently, the strained-Si gap is reduced as well as the electron conductivity mass as compared to bulk as a lower value, leading to an enhancement of the electron mobility by a factor of $2[28,29]$. Since intervalley carrier scattering may only occur between degenerate minima, electrons in a layer of (tensile)-strained silicon would undergo a lower number of intervalley scattering events per unit time than in bulk silicon. The combination of the effect pointed earlier makes tensile strained silicon devices excellent candidates to build the high-mobility FET channel that is necessary to detect $\mathrm{THz}$ radiation. The energy band diagram at zero voltage is presented in Figure 1(b) [26]. The value of the conduction band offset of the heterojunction $\mathrm{Si} / \mathrm{Si}_{0.70} \mathrm{Ge}_{0.30}$ is about $180 \mathrm{meV}$, ensuring an excellent electron confinement in the strained-Si quantum well layer that is necessary for room-temperature high-mobility operation of the detector. Table 1 summarizes the geometrical parameters and the value of the threshold voltage of the strained-Si MODFETs under study.

The channel's length $\left(\mathrm{L}_{\mathrm{DS}}\right)$ and width $\left(\mathrm{W}_{\mathrm{G}}\right)$ were kept constant for all devices $\left(\mathrm{L}_{\mathrm{DS}}=2 \mu \mathrm{m}\right.$, $\left.\mathrm{W}_{\mathrm{G}}=30 \mu \mathrm{m}\right)$. However, the gate lengths of the transistors were varied. Transistors with 100-, 250-, and 500-nm gate lengths were characterized. The gates were asymmetrically placed between the source (S) and the drain (D) contacts in all transistors; the distance between the right edge of the source and the left edge of the gate $\left(\mathrm{L}_{\mathrm{GS}}\right)$ was equal to $1 \mu \mathrm{m}$ for all the transistors (Table 1). An asymmetrical position of the gate is of interest to enhance THz detection by the transistor [30]. Measuring devices with different values of the gate length allows the study of the influence of the gate length on the performance of the transistors as $\mathrm{THz}$

\begin{tabular}{llllll}
\hline & $\mathrm{L}_{\mathrm{DS}}(\mu \mathrm{m})$ & $\mathrm{L}_{\mathrm{GS}}(\mu \mathrm{m})$ & $\mathrm{L}_{\mathrm{G}}(\mathrm{nm})$ & $\mathrm{W}_{\mathrm{G}}(\mu \mathrm{m})$ & $\mathrm{V}_{\mathrm{th}}(\mathrm{V})$ \\
\hline Device 1 (D1) & 2 & 1 & 100 & 30 & -0.75 \\
Device 2 (D2) & 2 & 1 & 250 & 30 & -0.67 \\
Device 3 (D3) & 2 & 1 & 500 & 30 & -0.62 \\
\hline
\end{tabular}

Table 1. Geometrical and electrical parameters of the strained-Si MODFETs under study.
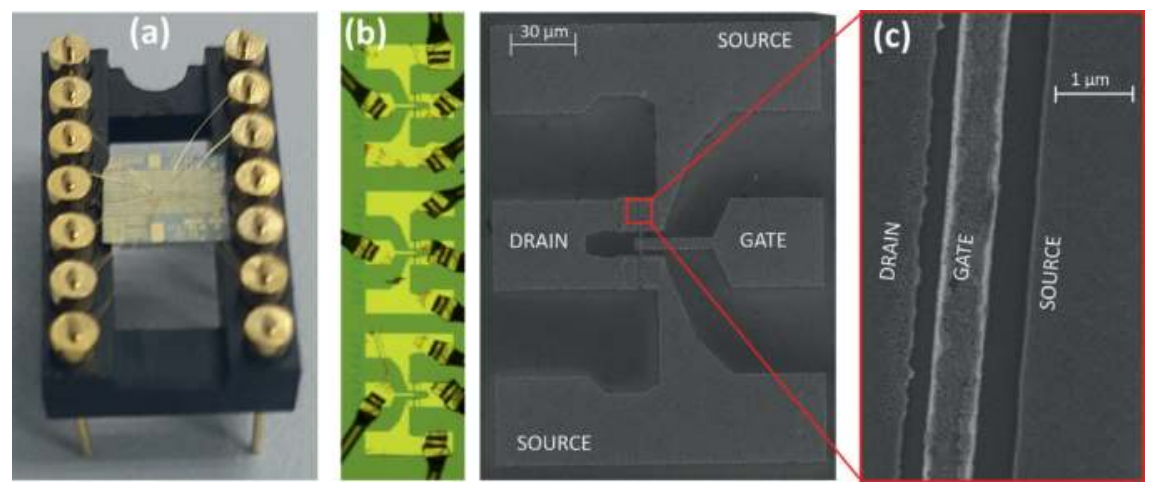

Figure 2. Strained-Si MODFETs under study mounted and bounded on a DIP14 (a) and their optical microscope image (b). (c) SEM image of device 3 (500-nm T-gate transistor). 
detectors and on the coupling of the incoming THz radiation. Strained-Si MODFETs were mounted and wire-bonded on the same dual in-line package (DIP14) shown in Figure 2(a). An optical microscope image of the three different devices under study is given in Figure 2(b). A SEM image of D3 with $\mathrm{L}_{\mathrm{G}}=500 \mathrm{~nm}$ is shown in Figure 2(c).

\subsection{TCAD modeling}

Dyakonov and Shur obtained an analytical solution of the unidimensional Euler equation that demonstrated the ability of the plasma waves in FET channels [17-19] to generate and detect $\mathrm{THz}$ radiation. A single equation cannot account for important parameters (such as doping profiles, high electric fields that locally modify the carrier mobility, device geometry, etc.) that condition the performance of the FET as a THz detector.

A better description of the charge transport in a transistor may be achieved through the numerical solution of the drift-diffusion model (DDM) that consists of the Poisson equation (Eq. (1)) and the continuity equations for electrons (Eq. (2)) and holes (Eq. (3)) [31]:

$$
\begin{gathered}
\nabla^{2} \varphi=-\frac{q}{\varepsilon}\left(p-n+N_{D}^{+}-N_{A}^{-}\right) \\
\frac{\partial n}{\partial t}=\frac{1}{q}\left(\vec{\nabla} \cdot \vec{J}_{n}\right)-U_{n} \\
\frac{\partial p}{\partial t}=-\frac{1}{q}\left(\vec{\nabla} \cdot \vec{J}_{p}\right)-U_{p}
\end{gathered}
$$

where $\phi$ is the electric potential, $q$ is the absolute value of the electron charge, $n / p$ is the electron/hole concentration, $\mathrm{N}_{\mathrm{D}}^{+}\left(\mathrm{N}_{\mathrm{A}}^{-}\right)$is the ionized donor (acceptor) concentration, $\varepsilon$ is the local material permittivity, and $U_{n}\left(U_{p}\right)$ represents the net electron (hole) recombination rate. $\vec{J}_{n}\left(\vec{J}_{p}\right)$ is the current density of electrons (holes) in the drift-diffusion model given by the following equations:

$$
\begin{aligned}
& \vec{J}_{n}=q \mu_{n}\left(u_{n}\right)\left[n \vec{E}+\vec{\nabla}\left(u_{n} n\right)\right] \\
& \vec{J}_{p}=q \mu_{p}\left(u_{p}\right)\left[n \vec{E}+\vec{\nabla}\left(u_{p} p\right)\right]
\end{aligned}
$$

where $\vec{E}$ is the electric field, $\mu_{n}\left(\mu_{p}\right)$ is the electron (hole) mobility, and $u_{n}\left(u_{p}\right)$ is the electron (hole) thermal voltage. In deep-submicron FETs, the drain and gate biases give rise to large electric fields that rapidly change over small length scales giving leading to nonlocal phenomena that dominate the transistor performance [26, 27]. As carriers are intensely heated by the electric field in the channel of deep-submicrometer FETs, energy balance equations accounting for electron and hole heating and energy relaxation in the device must be self-consistently added to the transport model. The DDM only considers moment relaxation [32], and therefore it is unable to describe a hot carrier transport. As channel mobility is closely dependent on the 
carrier temperature, an extended model needs to be used to study the electric properties of deep-submicron FET transistors used in plasma wave THz detection. This extended model is known as the hydrodynamic model (HDM).

The HDM $[32,33]$ includes a carrier energy balance by coupling to the set of DDM equations and the electron and hole energy flow densities that are given as follows:

$$
\begin{aligned}
& \vec{\nabla} \cdot \vec{S}_{n}=\frac{1}{q} \vec{J}_{n} \cdot \vec{E}-\frac{3}{2}\left(n \frac{u_{n}-u_{0}}{\tau_{n}}+\frac{\partial\left(u_{n} n\right)}{\partial t}\right) \\
& \vec{\nabla} \cdot \vec{S}_{p}=\frac{1}{q} \vec{J}_{p} \cdot \vec{E}-\frac{3}{2}\left(p \frac{u_{p}-u_{0}}{\tau_{p}}+\frac{\partial\left(u_{p} p\right)}{\partial t}\right)
\end{aligned}
$$

where $\vec{S}_{n}\left(\vec{S}_{p}\right)$ is the electron (hole) energy relaxation time, $\mathrm{u}_{\mathrm{n}}\left(\mathrm{u}_{\mathrm{p}}\right)$ is the electron (hole) thermal voltage, and the electric field that is self-consistently obtained from the Poisson equation.

Strained-Si MODFET is essentially a majority carrier device; then the hole energy balance equation (Eq. (7)) was disregarded in the model. In this work, a two-dimensional HDM (Eqs. (1)-(6)) was used. It was implemented with Synopsys TCAD [34]. Carrier relaxation times were obtained from uniform-field Monte Carlo simulations [35, 36]. In TCAD simulations, impurity de-ionization, Fermi-Dirac statistics, and mobility degradation due to both longitudinal and transverse electric field were considered. All TCAD simulations were carried out at room temperature.

The geometry and dimensions used in the simulations are the ones shown in Figure 1(a). The doping level of the supply layers was $10^{19} \mathrm{~cm}^{-3}$ for the upper supply layer and $1.8 \times 10^{18} \mathrm{~cm}^{-3}$ for the lower one. The thicknesses of the virtual substrate and the p-Si wafer were chosen to be 600 and $500 \mathrm{~nm}$, respectively, to economize computer memory. A uniform residual n-type-doping density of $10^{15} \mathrm{~cm}^{-3}$ was assumed in the non-intentionally doped regions of the transistor. Under both source and drain contacts, highly doped regions were considered to ensure ensure low values of contact resistance for the ohmic contacts of the device. The values of the conduction and valence bands offsets between the strained-Si and the relaxed $\mathrm{Si}_{1-\mathrm{x}} \mathrm{Ge}_{x}$ as a function of the Ge molar fraction $(\mathrm{x}=0.30)$ were extracted from [37]. Low electric field mobility in the channel was modeled using Roldan's model for biaxially strained Si on relaxed SiGe [38], and the maximum value electron mobility in the channel was $1600 \mathrm{~cm}^{2} /(\mathrm{Vs})$.

\section{THz setup}

Static (DC) and THz measurements were carried out at room temperature. On-wafer, DC measurements of drain-to-source current versus drain-to-source and gate-to-source bias voltages were done using a Cascade 11000B probe station and an Agilent B1500A semiconductor parameter analyzer. Figure 3 shows a photograph with a schematic of the experimental setup used for the terahertz characterization of the strained-Si MODFETs. A solid-state harmonic generator $\mathrm{THz}$ source based on a dielectric resonator oscillator (DRO) at $12 \mathrm{GHz}$ and electronic 


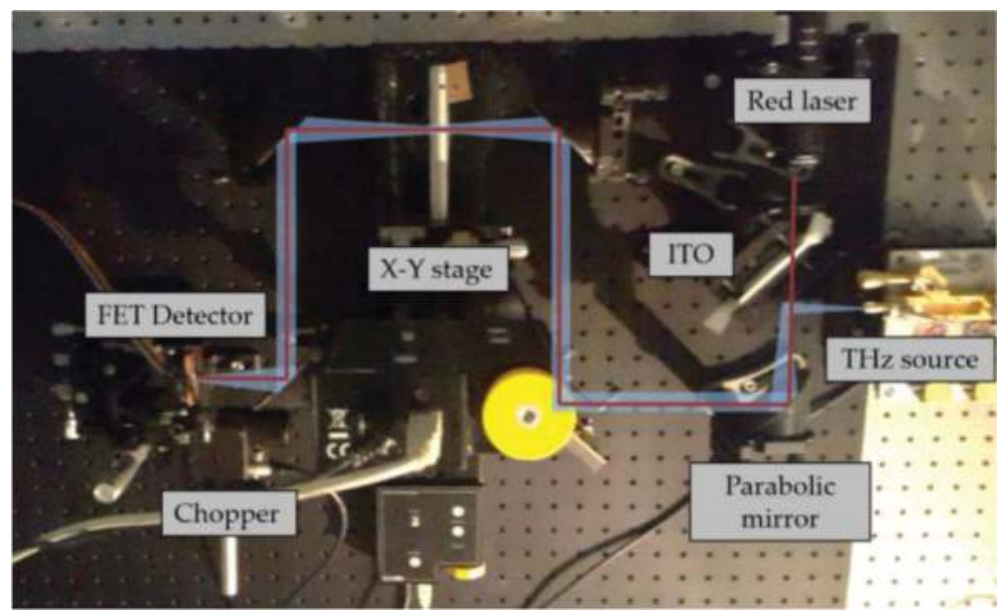

Figure 3. Photograph of the experimental setup and schematic description of the THz (blue) and red laser (red line) beams.

multiplication to reach $0.15 \mathrm{THz}$ with a power of $3 \mathrm{~mW}$ and $0.3 \mathrm{THz}$ with a power of $6 \mathrm{~mW}$ was used to excite the transistors. The output power was measured close to the source using a highly sensitive calibrated pyroelectric detector. The incoming $\mathrm{THz}$ radiation was modulated by a mechanical chopper between 0.233 and $5 \mathrm{kHz}$, collimated and focused by an indium tin oxide (ITO) mirror and off-axis parabolic and plane mirrors. A red LED (or laser) was used for the alignment of the $\mathrm{THz}$ beams.

The photo-induced drain-to-source voltage, $\Delta \mathrm{U}$, was measured using a lock-in technique. Finally, a X-Y stage was used to generate pixel-by-pixel THz images.

\section{Results and discussion}

\subsection{DC characterization}

Transfer characteristics of Device 3 are shown in Figure 4(a) for two values, 20 and $200 \mathrm{mV}$, of the drain-to-source voltage $\left(\mathrm{V}_{\mathrm{DS}}\right)$. The three transistors are depletion-mode devices, so a negative bias voltage must be applied to the gate (i.e., a negative gate-to-source voltage) to cut off the $c$ hannel $[25,39]$. Transfer characteristics in a log scale show that a total switch-off of the device was not possible, and a constant level of drain current $\left(\mathrm{I}_{\mathrm{DS}}\right)$ persists for a gate bias of $-1 \mathrm{~V}$ $\left(8 \mu \mathrm{A}\right.$ for $\mathrm{V}_{\mathrm{ds}}=20 \mathrm{mV}$ and $80 \mu \mathrm{A}$ for $\left.\mathrm{V}_{\mathrm{ds}}=200 \mathrm{mV}\right)$. As the drain voltage is moderately raised from 20 to $200 \mathrm{mV}$, the above-described behavior is enhanced, and the sub-threshold current at $V_{G S}=-1 \mathrm{~V}$ increases when $V_{D S}$ increases. As pointed out earlier, this behavior reveals a moderate control of the channel by the gate electrode due to the double supply layer; in return, this double deck ensures a suitable concentration of the electron plasma in the channel that is of paramount importance to achieve a good performance of the transistor in $\mathrm{THz}$ detection. 
(a)

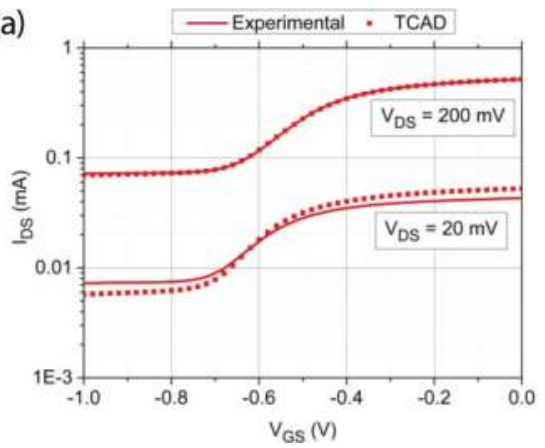

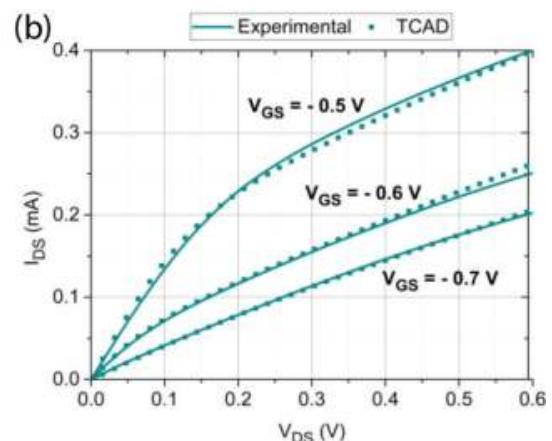

Figure 4. (a) Experimental and simulated transfer characteristics for device 3 for two values of the drain voltage plotted in a $\log$ scale and (b) output characteristics for three different values of $\mathrm{V}_{\mathrm{GS}}$.

The TCAD simulation model of the transistor was validated through comparison with DC and AC measurements. The agreement between TCAD and experimental results across the whole ranges of gate-to-source and drain-to-source biases studied is excellent as shown in Figure 4.

Agreement between measurement and simulation magnitudes involving first derivatives of the drain current was also analyzed. In the first place, the efficiency of the transconductance was analyzed. This magnitude defined as the ratio of transconductance $\left(\mathrm{g}_{\mathrm{m}}\right)$ to drain-tosource DC current $\left(\mathrm{I}_{\mathrm{DS}}\right)$, is a key parameter used to compare the performance of different technologies of transistors. The efficiency of the transconductance is used here, on the one hand, because it clearly shows the operation region of the device and, on the other hand, because the efficiency of the transconductance is linearly dependent on a current derivative and discrepancies between simulation and experimental results are readily revealed. Figure 5(a) gives the experimental and calculated efficiency of the transconductance versus the gate voltage $\left(\mathrm{V}_{\mathrm{GS}}\right)$ of the device D3. The maximum value of the efficiency of the transconductance measured
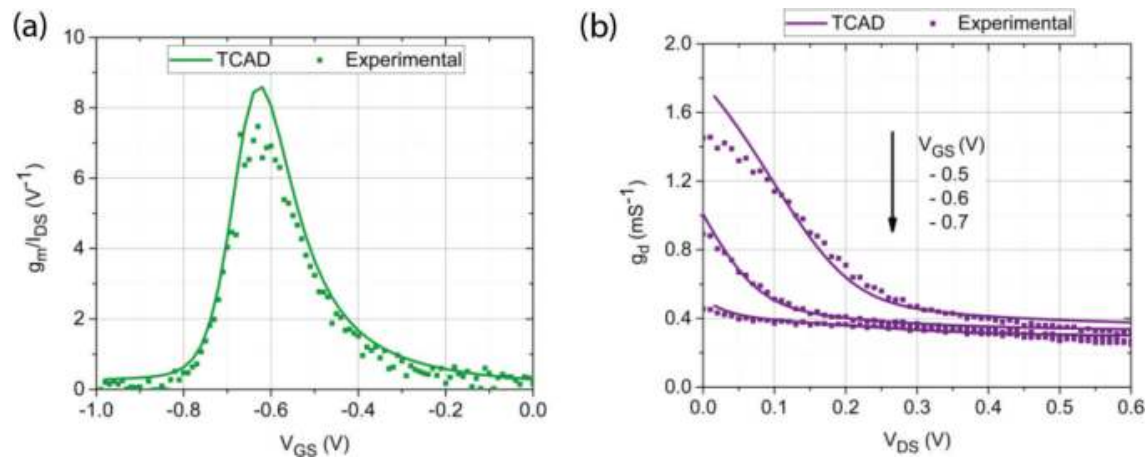

Figure 5. (a) Efficiency of the transconductance versus the gate voltage obtained from measurements and numerical TCAD simulations and (b) drain conductance versus drain voltage for three different values of gate bias obtained from measurements and numerical TCAD simulations. 
and obtained from TCAD simulations $\left(<10 \mathrm{~V}^{-1}\right)$ is significantly lower than the theoretical limit $\left(38 \mathrm{~V}^{-1}\right)$, along with the above-discussed behavior of the transconductance, suggesting that further improvements of the transistor performance may be achieved by an optimization of the layout of the structure.

In the second place, the drain conductance that gives the first derivative of the drain current with respect to the drain current was also determined. Figure 5(b) shows drain conductance as a function of drain voltage for three different gate voltages $(-0.5,-0.6$, and $-0.7 \mathrm{~V})$. Experimental and TCAD drain conductance curves show an excellent agreement between measurements and TCAD simulations.

\subsection{THz detection: TCAD versus experimental}

A TCAD study of the $\mathrm{THz}$ photovoltaic response of the transistor was implemented, as in measurements, grounding the source, biasing the gate, and floating the drain contact while a $\mathrm{THz}$ small sinusoidal signal $(0.15$ or $0.3 \mathrm{THz})$ was superimposed to the gate voltage as described in [17-19]. As the DC drain voltage setup in the photovoltaic mode must be supported by a net charge in the drain region, in TCAD simulations, a charge boundary condition was implemented at the floating drain contact with a distributed boundary condition over all nodes of the mesh of the drain electrode. The boundary condition is as follows:

$$
\oint \vec{D} \cdot \overrightarrow{d S}=Q
$$

where $\vec{D}$ is the electric displacement field, Q is the total net charge, and the integral is evaluated over the entire surface of the drain electrode. Eq. (8) forces the potential on the drain (i.e., the photoresponse of the detector) to be adjusted to produce the correct total charge on the electrode. HDM equations (Eqs. (1)-(6)) were solved in the time domain to obtain the transistor photoresponse. The amplitude of the sinusoidal signal superimposed in the gate contact was fixed to an arbitrary value of $5 \mathrm{mV}$. Since this value is arbitrary, the magnitude of the $\mathrm{THz}$ response obtained in simulations will be presented henceforward as arbitrary magnitude in figures.

In TCAD simulations, it was found that the drain voltage $(\Delta \mathrm{U})$ induced by the THz sinusoidal signal exhibits both the same shape (sinusoidal) and the frequency than the AC signal superimposed to the gate bias; this ensures that no frequency conversion takes place in the simulated devices. In addition, it was found that its amplitude is considerably smaller than one of the gate's signal in agreement with the fact that in the $\mathrm{THz}$ range the transistor is unable to amplify signals and it is merely working as a $\mathrm{THz}$ detector. The mean value of the induced drain voltage by the radiation was negative as predicted by theoretical models [17-19]. The photoresponse obtained in TCAD simulations was extracted by subtracting the value of drain-to-source voltage when the THz signal was applied $\left(\Delta U_{T H z-o n}\right)$ from the drainto-source voltage when no signal was applied $\left(\Delta U_{T H z-o f f}\right)$ :

$$
\Delta U=\Delta U_{T H z-o f f}-\Delta U_{T H z-o n}
$$


(a)

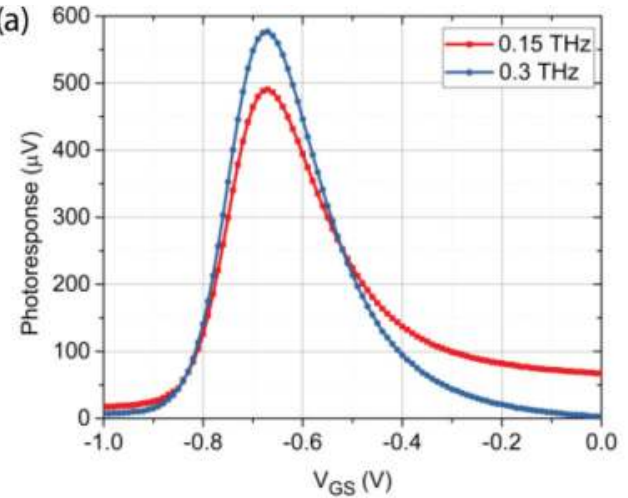

(b)

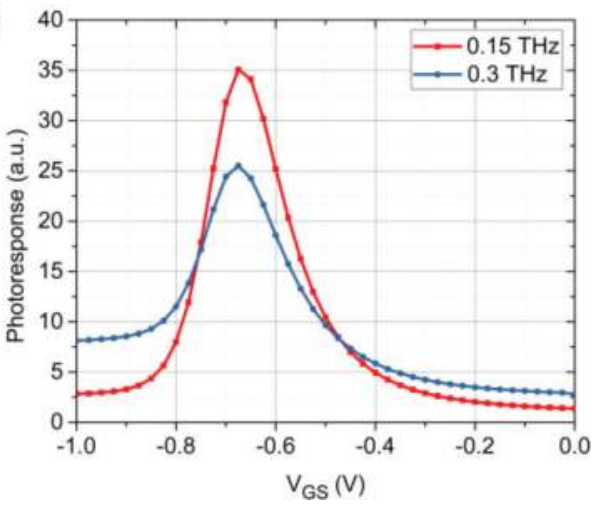

Figure 6. Measured (a) and TCAD simulation (b) photoresponse versus gate voltage under excitation of 0.3 (blue dots) and $0.15 \mathrm{THz}$ (red squares) of D2.

Figure 6 gives the room-temperature photovoltaic response of device D2 with $\mathrm{L}_{\mathrm{G}}=250 \mathrm{~nm}$ obtained experimentally and from TCAD simulations at 0.3 (blue squares) and $0.15 \mathrm{THz}$ (red dots).

The higher value in the photoresponse was found when the gate electrode was voltage-biased at a voltage close to the threshold voltage of the transistor [40]. This behavior has been observed earlier in FETs $[16,20]$ and it was attributed to a non-resonant (broadband) response of the detector. It is related to over-damping of the plasma waves in the channel where the AC current generated by the incoming radiation at the source cannot reach the drain side of the channel. A theoretical study of the photoresponse in this regime is presented in [21, 22]. The quality factor [19] is given by: $Q=\omega \tau$, where $\tau$ is the relaxation time given by $\mathrm{m}^{*} \mu / \mathrm{e}\left(\mathrm{m}^{*}\right.$ : the electron effective mass, $\mu$ : the electron mobility, and e: the absolute value of the electron charge). In the present case, the devices show a higher channel mobility $\left(\sim 1600 \mathrm{~cm}^{2} / \mathrm{V} \cdot \mathrm{s}\right)$ as compared to the conventional Si-MOSFET ( $\left.\sim 200 \mathrm{~cm}^{2} / \mathrm{V} . \mathrm{s}\right)$; the value of the quality factor was estimated to be 0.14 at $f=0.15 \mathrm{THz}$ and $\sim 0.29$ at $f=0.3 \mathrm{THz}$; any of these values fulfills the resonance condition. The experimental photoresponse (Figure 6(a)) is more intense under excitation at $0.3 \mathrm{THz}$ than at $0.15 \mathrm{THz}$. The photoresponse obtained in TCAD simulations exhibits the opposite behavior: the photoresponse at $0.15 \mathrm{THz}$ is more intense than at $0.3 \mathrm{THz}$. This must be partly attributed to the fact that in measurements, the source's output power at $0.3 \mathrm{THz}$ is twice than at $0.15 \mathrm{THz}$. Moreover, the coupling of the $\mathrm{THz}$ radiation to the devices could vary at 0.15 and $0.3 \mathrm{THz}$. These possibilities will be explored and further discussed subsequently. As in TCAD simulations, the amplitude of the sinusoidal gate signal was fixed to $5 \mathrm{mV}$ for both frequencies, and no coupling and/or effects related to the differences in the incoming $\mathrm{THz}$ power at both frequencies can be found.

Besides the photovoltaic mode, the efficiency of the detector can be improved, creating additional asymmetries between the drain and the source [30, 41, 42]. One method to generate these asymmetries is to apply a DC current between the drain and the source $\left(\mathrm{I}_{\mathrm{DS}}>0\right)$. Figure 7 shows the photoresponse obtained experimentally and from TCAD simulations when a drain-to-source current bias, $\mathrm{I}_{\mathrm{DS}}=50 \mu \mathrm{A}$, is imposed to the transistor D1 at 0.15 and at $0.3 \mathrm{THz}$. 

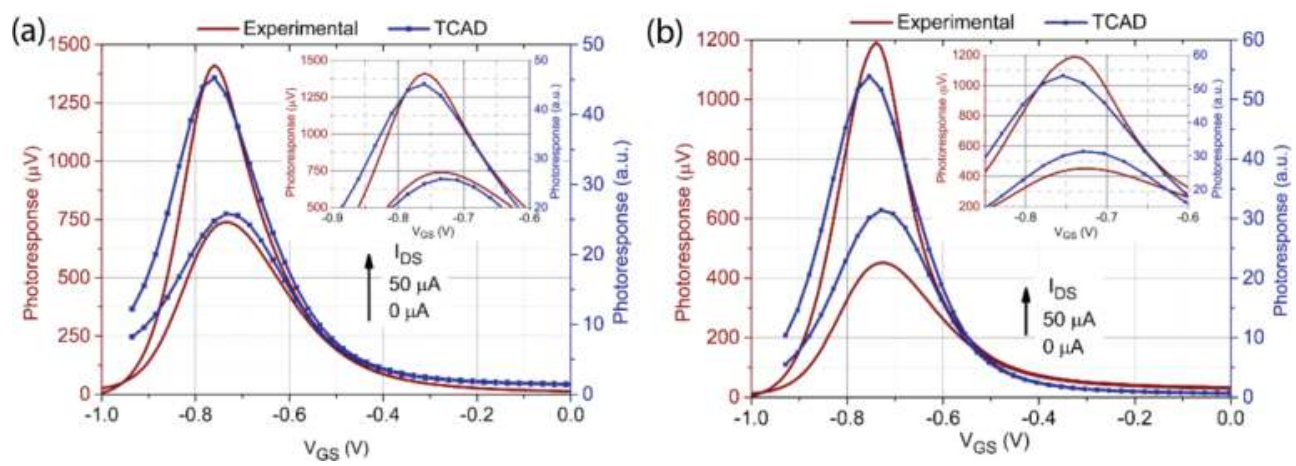

Figure 7. Photoresponse versus gate voltage measured experimentally (red color) and obtained from TCAD simulations (blue color) for different values of $\mathrm{I}_{\mathrm{DS}}$ at 0.3 (a) and $0.15 \mathrm{THz}$ (b) for D1. The insets show a zoom of the region of maximum photoresponse.

The main effect of imposing a DC source-to-drain current bias is that THz detection is significantly enhanced, and the photoresponse grows when the bias current is increased. The bias current introduces an additional asymmetry between the source and the drain, creating a depletion of the electron density on the drain side of the channel, and consequently, the maximum value of the photoresponse is increased [24, 41, 42]. Moreover, a noticeable additional effect (the insets in Figure 7) is that the maximum in the photoresponse is shifted toward more negative values of the gate voltage. Figures 6 and 7 show that the MODFET photoresponse exhibits the same dependence with respect to the gate bias voltage both in measurements and in simulations. An excellent agreement between TCAD and experimental results is found in the photovoltaic mode $\left(I_{D S}=0\right)$ and even when a bias current $\left(I_{D S}\right)$ is applied to the transistor. Therefore, the measured photoresponse of the strained-Si MODFET must be mainly attributed to the plasmonic response of the channel carriers rather than to the antenna role played by bonding wires or metal pads as simulations reproduce correctly the experimental photoresponse.

In addition, to the abovementioned channel asymmetry created by the current bias, a built-in asymmetry can be introduced geometrically by imposing an asymmetric design of the contact pads of the transistor. Figure 8 shows the obtained photoresponse from TCAD simulations as a function of the asymmetry factor. The latter is defined as the ratio $\mathrm{L}_{\mathrm{GS}} / \mathrm{L}_{\mathrm{GD}^{\prime}}$, where $\mathrm{L}_{\mathrm{GS}}$ is the distance between the right edge of the source and the left edge of the gate and $\mathrm{L}_{\mathrm{GD}}$ is the distance between the right edge of the gate and the left edge of the drain (Figure 1(a)). $\mathrm{L}_{\mathrm{GS}} / \mathrm{L}_{\mathrm{GD}}=1$ means that the transistor is symmetric. The gate length was kept constant at $500 \mathrm{~nm}$ for all the transistors.

A higher photoresponse signal was obtained for an asymmetry factor of 0.2 where the gate finger is very close to the source contact. However, in the opposite case, when the finger is close to the drain pad, no enhancement of the photoresponse was obtained. In the non-resonant regime, the oscillation of the plasma occurs close to the source pad where the electrons are injected into the channel and hence the gate finger close to the source pad could control efficiently the damped oscillation of the plasma waves. We conclude that for a better performance, asymmetry should be introduced as close as possible to the source pad. 


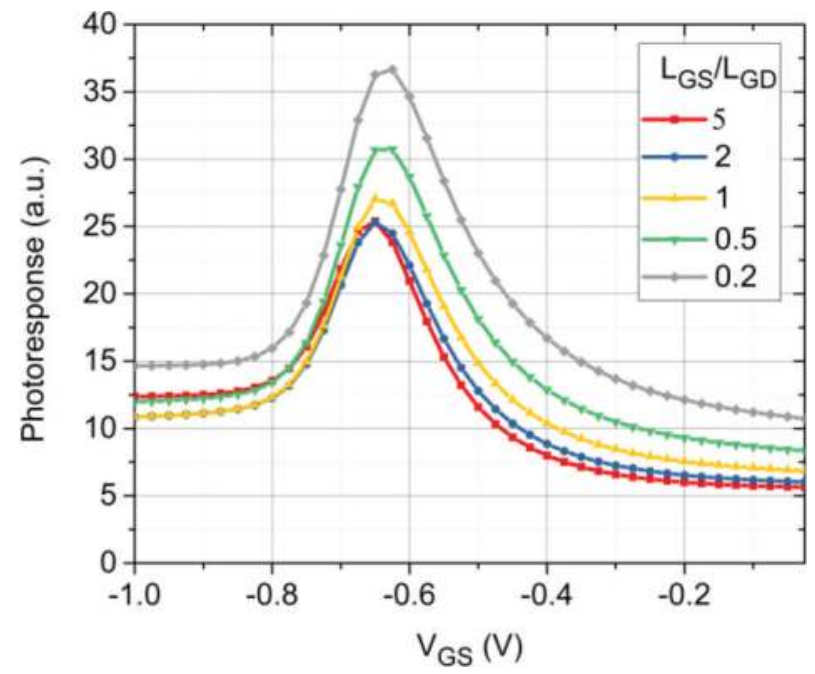

Figure 8. Photoresponse obtained from TCAD simulations versus $\mathrm{V}_{\mathrm{GS}}$ for different horizontal positions of the gate contact.

\subsection{Polarization sensitivity of photoresponse}

It can be observed in Figures 6(a) and 7 that the obtained photoresponse is more intense under excitation at 0.3 than at $0.15 \mathrm{THz}$. This must be partly attributed to the higher power at $0.3 \mathrm{THz}(\sim 6 \mathrm{~mW})$ than at $0.15 \mathrm{THz}(\sim 3 \mathrm{~mW})$ and to the coupling of the $\mathrm{THz}$ radiation to the device that varies with frequency. Moreover, the bonding wires and the metallic pads could play an antenna role to couple the incoming terahertz radiation (linearly polarized) to the 2D electron channel [43-45]. To understand how radiation is coupled, devices were rotated in the plane perpendicular to the terahertz beam, and the photoresponse signal was measured for

(a)

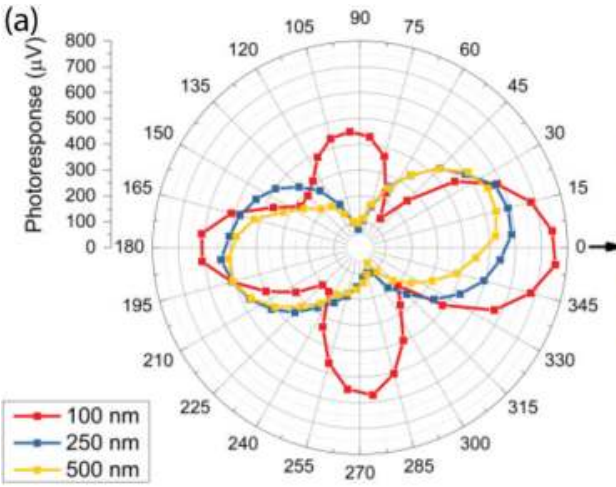

(b)

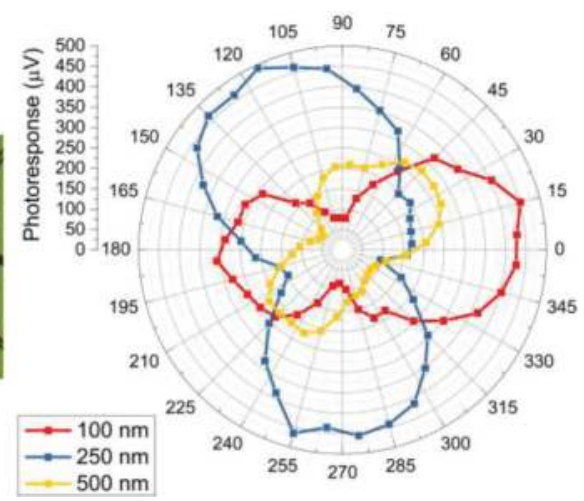

Figure 9. Photoresponse versus rotation angle for all devices under excitation of 0.3 (a) and $0.15 \mathrm{THz}$ (b). The inset figure shows the devices at the zero-angle position. 
each angular position of the devices. Figure 9 shows the photoresponse signal as a function of the polarization of the incoming $\mathrm{THz}$ radiation for 0.3 (a) and $0.15 \mathrm{THz}(\mathrm{b})$.

For all the devices at $0.3 \mathrm{THz}$, a maximum of the photoresponse signal was found when the incoming radiation was parallel to the gate finger pads (see the inset of Figure 9(a) at $0^{\circ}$ ), showing a maximum photoresponse for all the devices at the same angular position. At 0.15 $\mathrm{THz}$ the maximum photoresponse was obtained at different angular positions for each device. It is clearly shown that at a lower frequency $(0.15 \mathrm{THz})$, bounding wires play an important role to couple the terahertz radiation to the channel of the device. Moreover, at a higher frequency $(0.3 \mathrm{THz})$, the coupling is performed by the contact pads and/or the gate fingers. These results are in agreement with previously published ones [46].

\subsection{Responsivity, NEP, and imaging}

Responsivity $\left(R_{V}\right)$ and noise equivalent power (NEP) are the two key parameters (figures of merit) that determine the performance of $\mathrm{THz}$ detectors. Responsivity is calculated according to the expression:

$$
R_{V}=\frac{\Delta \mathrm{US}_{\mathrm{t}}}{\mathrm{P}_{\mathrm{t}} \mathrm{S}_{\mathrm{a}}} \frac{\pi}{\sqrt{2}}
$$

where $\Delta \mathrm{U}$ is the photoresponse signal measured with the lock-in amplifier, $S_{t}$ is the radiation beam spot area, $S_{a}$ is the active area of the transistor, and $P_{t}$ is the total incident power surrounding the detector. The radiation beam power and spot area were measured using a calibrated pyroelectric detector at the MODFET position (see Figure 3); the $P_{t}$ values were $P_{t}=0.5 \mathrm{~mW}$ at $0.15 \mathrm{THz}$ and $P_{t}=1 \mathrm{~mW}$ at $0.3 \mathrm{THz}$. The spot area is given by $\pi r^{2}$ where $r$ is the radius of the beam spot $(\approx 1.5 \mathrm{~mm}$ at $0.3 \mathrm{THz}$ and $3.3 \mathrm{~mm}$ at $0.15 \mathrm{THz})$. The area of each single transistor, including the contact pads, is less than $0.05 \mathrm{~mm}^{2}$ (Figure 2), that is, it is much smaller than the diffraction limit area $S_{\lambda}=\lambda^{2} / 4$. Accordingly, to calculate $R v$ in Eq. (6), $S a$ was replaced by $S_{\lambda}$ to avoid overestimation of the $R v$ as well as NEP. The factor $\pi / \sqrt{2}$ originates from the Fourier transform of the square wave-modulated THz signal detected as RMS value with a lock-in.

The NEP is given by $N_{t h} / R_{V}$, where $N_{t h}$ is the thermal noise of the transistor in $\mathrm{V} / \mathrm{Hz}^{0.5}$ and $R_{V}$ is the responsivity in $\mathrm{V} / \mathrm{W}$. Since $R_{V}$ and the NEP were studied at zero drain current bias, the thermal noise $N_{t h}=\left(4 k T R_{d s}\right)^{0.5}$ is the only relevant source of noise of the transistor. Here, $R_{d s}$ is the drain-to-source resistance that can be extracted from the transfer characteristics measured at a low drain bias $(20 \mathrm{mV})$ corresponding to the linear regime (i.e., Figure 5(a)).

Figure 10 presents the responsivity and NEP curves for D1 with $\mathrm{L}_{\mathrm{G}}=100 \mathrm{~nm}$ at 0.15 and 0.3 THz. Table 2 summarizes the obtained NEP and Rv for the Si-MODFETs at 0.15 and at $0.3 \mathrm{THz}$. Device 1, with the shorter gate, exhibits the best performance at $0.3 \mathrm{THz}$ with $\mathrm{R}_{\mathrm{v}}=46.4 \mathrm{~V} / \mathrm{W}$ and NEP $\sim 0.12 \mathrm{nW} / \mathrm{Hz}^{0.5}$ and Device 2 exhibits the best performance at $0.15 \mathrm{THz}$ with $\mathrm{R}_{\mathrm{V}}=74.5 \mathrm{~V} / \mathrm{W}$ and NEP $\sim 0.06 \mathrm{nW} / \mathrm{Hz}^{0.5}$. This must be attributed to the large photoresponse signal provided by the Si/SiGe MODFET and to a better coupling of the incoming terahertz radiation. The values obtained for the NEP and the responsivity are comparable to 

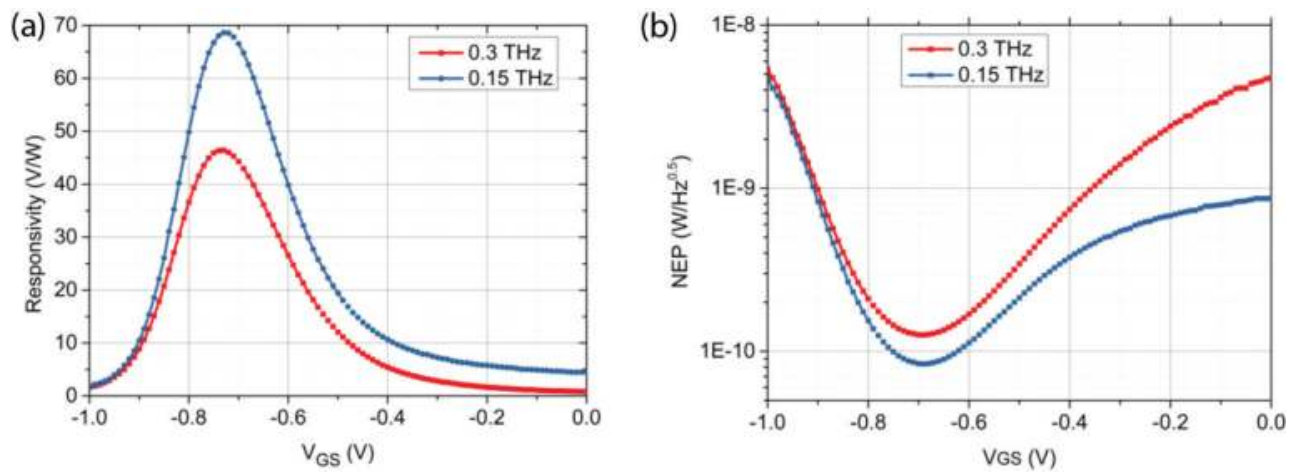

Figure 10. Responsivity (a) and NEP (b) measured under excitation of 0.15 (blue dots) and $0.3 \mathrm{THz}$ (red squares) of the strained-Si MODFET with 100-nm gate length.

\begin{tabular}{lllll}
\hline & $\mathbf{0 . 1 5} \mathbf{T H z}$ & & $\mathbf{0 . 3} \mathbf{T H z}$ \\
\cline { 2 - 5 } & $\mathrm{R}_{\mathrm{v}}(\mathrm{V} / \mathrm{W})$ & $\mathbf{N E P}(\mathrm{nW} / \sqrt{\mathrm{Hz}})$ & $\mathrm{R}_{\mathrm{v}}(\mathrm{V} / \mathrm{W})$ & $\mathbf{N E P}(\mathrm{nW} / \sqrt{\mathrm{Hz}})$ \\
\hline Device 1 & 68.6 & 0.08 & 46.4 & 0.12 \\
Device 2 & 74.5 & 0.06 & 36.2 & 0.13 \\
Device 3 & 41.1 & 0.12 & 33.3 & 0.14 \\
\hline
\end{tabular}

Table 2. Calculated NEPs and $R_{v}$ for the different devices under studio.

the ones of commercial terahertz detectors at room temperature like Golay cells, pyroelectric detectors, and Schottky diodes [47]. However, the Si/SiGe MODFET presents the advantage of working at higher modulation frequencies as compared to other detectors.

To test the ability of the strained-Si MODFETs as detectors in THz imaging, a single transistor (D1) was used as the sensor in the terahertz imaging system shown in Figure 3. Figure 11 shows the visible image of a standard copper RT/duroid ${ }^{\circledR}$ laminate where the logo of the Nanotechnology Group at Salamanca University has been etched (a) and its terahertz image at $0.3 \mathrm{THz}(\mathrm{b})$ when it was wrapped around with a paper. $\mathrm{THz}$ radiation passes through in the regions were the metal layer was etched off and it is reflected in the regions covered with copper. A pixel-by-pixel image was taken using D1 as the detector; the gate of the transistor

(a)

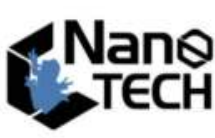

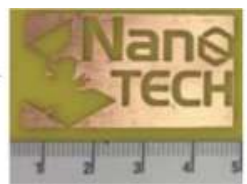

(b)

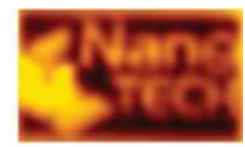

Figure 11. Visible (a) and $0.3 \mathrm{THz}(\mathrm{b})$ images obtained at room temperature using the strained-Si MODFET with a shorter gate as sensor. 
was biased around its threshold voltage to obtain a maximum intensity of the signal. The clear terahertz image obtained of the hidden object confirms the suitability of strained-Si MODFETs to be used as detectors to obtain high-quality $\mathrm{THz}$ images. Better resolution could be obtained at higher frequencies owing to its lower wavelength $(\lambda<1 \mathrm{~mm})$.

\section{Conclusions}

The potential of submicron gate length strained-Si MODFETs as detectors of terahertz radiation was demonstrated. A broadband (non-resonant) $\mathrm{THz}$ detection was observed under excitation of the transistors by a continuous-wave source at 0.15 and $0.3 \mathrm{THz}$. TCAD results obtained using a HDM model were in good agreement with the experimental ones in terms of both the excitation frequency and the gate-to-source bias. When imposing a source-todrain current of $50 \mu \mathrm{A}$, both TCAD simulations and experiments show an increase of the photoresponse as compared to the photovoltaic mode. A theoretical study was performed to analyze the effect of gate's geometrical asymmetries on the $\mathrm{THz}$ detection. Coupling between THz radiation and strain-Si MODFETs channel was analyzed at 0.3 and $0.15 \mathrm{THz}$. It shows that the coupling is mainly performed by bonding wires at $0.15 \mathrm{THz}$. Finally, the strained-Si MODFET was used as a single pixel detector to obtain images of a concealed object at $0.3 \mathrm{THz}$.

\section{Acknowledgements}

We would like to acknowledge Thomas Hackbarth (Daimler AG) who fabricated the strainedSi MODFETs used in this work. Our work was financially supported by the Spanish Ministry of Economy and Commerce and FEDER (ERDF: European Regional Development Fund) under the Research Grant \#TEC2015-65477-R and FEDER/Junta de Castilla y León Research Grant \#SA045U16. Both Research Grants \#TEC2015-65477-R and Salamanca University partly support Open Source publications. Y. M. Meziani acknowledges the financial support from RIEC nation-wide Collaborative Reseach Project, Sendai, Japan.

\section{Author details}

Juan Antonio Delgado-Notario ${ }^{1 *}$, Vito Clericò ${ }^{1}$, Kristel Fobelets ${ }^{2}$, Jesús Enrique Velázquez-Pérez ${ }^{1}$ and Yahya Moubarak Meziani ${ }^{1}$

*Address all correspondence to: juanandn@usal.es

1 USAL NanoLab, University of Salamanca, Salamanca, Spain

2 Department of Electrical and Electronic Engineering, Imperial College, South Kensington Campus, London, UK 


\section{References}

[1] Aniel F, Enciso-Aguilar M, Giguerre L, Crozat P, Adde R, Mack T, Seiler U, Hackbarth T, Herzog HJ, König U, Raynor B. High performance $100 \mathrm{~nm}$ T-gate strained Si/Si0.6Ge0.4 n-MODFET. Solid-State Electronics. 2003;47:283-289. DOI: 10.1016/S0038-1101(02)00208-3

[2] Rainsford T, Mickan SP and Abbott D. In T-Ray Sensing Applications: Review of Global Developments, Conference on Smart Structures, Devices, and Systems II; 13-15 December 2004; Sydney, Australia, 2005. pp. 826-838. DOI: 10.1117/12.607746

[3] Wiedner MC, Wieching G, Bielau F, Rettenbacher K, Volgenau NH, Emprechtinger M, Graf UU, Honingh CE, Jacobs K, Vowinkel B, Menten KM, Nyman LÅ, Güsten R, Philipp S, Rabanus D, Stutzki J, Wyrowski F. First observations with CONDOR, a 1.5 THz heterodyne receiver. Astronomy and Astrophysics. 2006;454:L33. DOI: 10.1051/ 0004-6361:20065341

[4] Siegel PH. Thz instruments for space. IEEE Transactions on Antennas and Propagation. 2007;55:2957-2965. DOI: 10.1109/TAP.2007.908557

[5] Clericò V, Delgado-Notario JA, Campos N, Gómez D, Diez E, Velazquez JE, Meziani YM. Terahertz spectroscopy of a multilayers flake of graphene. Journal of Physics: Con ference Series. 2015;647:012040. DOI: 10.1088/1742-6596/647/1/012040

[6] Crowe TW, Globus T, Woolard DL, Hesler JL. Terahertz sources and detectors and their application to biological sensing. Philosophical Transactions of the Royal Society of London Series a-Mathematical Physical and Engineering Sciences. 2004;362:365-374. DOI: 10.1098/rsta.2003.1327

[7] Chen W, Peng Y, Jiang X, Zhao J, Zhao H, Zhu Y. Isomers identification of 2-hydroxyglutarate acid disodium salt $(2 \mathrm{hg})$ by terahertz time-domain spectroscopy. Scientific Reports. 2017;7:12166. DOI: 10.1038/s41598-017-11527-z

[8] Krimi S, Klier J, Jonuscheit J, Freymann G, Urbansky R, Beigang R. Highly accurate thickness measurement of multi-layered automotive paints using terahertz technology. Applied Physics Letters. 2016;109:021105. DOI: 10.1063/1.4955407

[9] Blin S, Teppe F, Tohme L, Hisatake S, Arakawa K, Nouvel P, Coquillat D, Penarier A, Torres J, Varani L, Knap W, Nagatsuma T. Plasma-wave detectors for terahertz wireless communication. IEEE Electron Device Letters. 2012;33:1354-1356. DOI: 10.1109/ LED.2012.2210022

[10] Federici JF, Schulkin B, Huang F, Gary D, Barat R, Oliveira F, Zimdars D. THz imaging and sensing for security applications - explosives, weapons and drugs. Semiconductor Science and Technology. 2005;20:S266-S280. DOI: 10.1088/0268-1242/20/7/018

[11] Kleine-Ostmann T. In Thz metrology, 38th international conference on infrared, millimeter, and terahertz waves (IRMMW-THz); 01-06 September 2013; Mainz, Germany. DOI: 10.1109/IRMMW-THz.2013.6665913 
[12] Ahi K, Shahbazmohamadi S, Asadizanjani N. Quality control and authentication of packaged integrated circuits using enhanced-spatial-resolution terahertz timedomain spectroscopy and imaging. Optics and Lasers in Engineering. 2017. DOI: 10.1016/j. optlaseng.2017.07.007

[13] Chan WL, Deibel J, Mittleman DM. Imaging with terahertz radiation. Reports on Progress in Physics. 2007;70:1325-1379. DOI: 10.1088/0034-4885/70/8/R02

[14] Wang M, Yang EH. THz applications of 2D materials: Graphene and beyond. NanoStructures \& Nano-Objects. 2017. DOI: 10.1016/j.nanoso.2017.08.011

[15] Hou HW, Liu Z, Teng JH, Placios T, Chua SJ. High temperature terahertz detectors realized by a GaN high electron mobility transistor. Scientific Reports. 2017;7:46664. DOI: 10.1038/srep46664

[16] Meziani YM et al. Non- resonant response to terahertz radiation by submicron cmos transistors. IEICE Transactions on Electronics. 2006;E89C:993-998. DOI: 10.1093/ietele/ e89-c.7.993

[17] Dyakonov M, Shur M. Shallow-water analogy for a ballistic field-effect transistor new mechanism of plasma-wave generation by dc current. Physical Review Letters. 1993;71:2465-2468. DOI: 10.1103/PhysRevLett.71.2465

[18] Dyakonov M, Shur M. 2-dimensional electronic flute. Applied Physics Letters. 1995;67:11371139. DOI: $10.1063 / 1.114986$

[19] Dyakonov M, Shur M. Detection, mixing, and frequency multiplication of terahertz radiation by two-dimensional electronic fluid. IEEE Transactions on Electron Devices. 1996;43:380-387. DOI: 10.1109/16.485650

[20] Knap W, Deng Y, Rumyantsev S, Shur M. Resonant detection of subterahertz and terahertz radiation by plasma waves in submicron field-effect transistors. Applied Physics Letters. 2002;81:4637-4639. DOI: 10.1063/1.1473685

[21] Delgado-Notario JA, Meziani YM, Velazquez-Perez JE, Fobelets K. Optimization of thz response of strained-si modfets. Physica Status Solidi C: Current Topics in Solid State Physics. 2015;12, 12(12):1401-1404. DOI: 10.1002/pssc.201510136

[22] Delgado-Notario JA, Javadi E, Calvo-Gallego J, Diez E, Velazquez JE, Meziani YM, Fobelets K. Sub-micron gate length field effect transistors as broad band detectors of terahertz radiation. Fundamental and Applied Problems of Terahertz Devices and Technologies. 2017;58:87-95. DOI: 10.1142/S0129156416400206

[23] Meziani YM, Garcia-Garcia E, Velazquez-Perez JE, Coquillat D, Dyakonova N, Knap W, Grigelionis I, Fobelets K. Terahertz imaging using strained-si modfets as sensors. Solid State Electronics. 2013;83:113-117. DOI: 10.1016/j.sse.2013.01.030

[24] Delgado-Notario JA, Meziani YM, Velazquez-Perez JE, Fobelets K. Sub-THz imaging using non-resonant HEMT detectors. Sensors. 2018;18:543. DOI: 10.3390/s18020543 
[25] Fobelets K, Jeamsaksiri W, Papavasilliou C, Vilches T, Gaspari V, Velazquez-Perez JE, Michelakis K, Hackbarth T, Konig U. Comparison of sub-micron Si:SiGe heterojunction nFETs to Si nMOSFET in present-day technologies. Solid State Electronics. 2004;48:14011406. DOI: 10.1016/j.sse.2004.01.017

[26] RumyantsevSL, FobeletsK, VekslerD, Hackbarth T, ShurM.Strained-si modulation doped field effect transistors as detectors of terahertz and sub-terahertz radiation. Semiconductor Science and Technology. 2008;23:105001. DOI: 10.1088/0268-1242/23/10/105001

[27] Gaspari V, Fobelets K, Velazquez-Perez JE, Hackbarth T. Dc performance of deep submicrometer schottky-gated n-channel Si:Sige hFETs at low temperature. IEEE Transactions on Electron Devices. 2005;52:2067-2074. DOI: 10.1109/TED.2005.855059

[28] Braunstein R, Moore AR, Herman F. Intrinsic optical absorption in germanium-silicon alloys. Physical Review. 1958;109:695-710. DOI: 10.1103/PhysRev.109.695

[29] Oberhüber R, Zandler G, Vogl P. Subband structure and mobility of two-dimensional holes in strained Si/SiGe MOSFETs. Physical Review B. 1998;58:9941-9947. DOI: 10.1103/ PhysRevB.58.9941

[30] Kurita Y, Ducournau G, Coquillat D, Satou A, Kobayashi K, Tombet SB, Meziani YM, Popov VV, Knap W, Suemitsu T, et al. Ultrahigh sensitive sub-terahertz detection by inpbased asymmetric dual-grating-gate high-electron-mobility transistors and their broadband characteristics. Applied Physics Letters. 2014;104:251114. DOI: 10.1063/1.4885499

[31] Hänsch W. The Drift Diffusion Equation and its Applications in MOSFET Modeling. Wien: Springer-Verlag; 1991. DOI: 10.1007/978-3-7091-9095-1

[32] Grasser T, Tang TW, Kosina H, Selberherr S. A review of hydrodynamic and energytransport models for semiconductor device simulation. Proceedings of the IEEE. 2003;91:251274. DOI: 10.1109/JPROC.2002.808150

[33] Meinerzhagen B, Engl WL. The influence of the thermal-equilibrium approximation on the accuracy of classical two-dimensional numerical modeling of silicon submicrometer mos-transistors. IEEE Transactions on Electron Devices. 1988;35:689-697. DOI: 10.1109/ 16.2514

[34] Taurus Medici: Taurus Taurus User Guide, Version X-2005. 10, Synopsys, Inc., Mountain View, California; 2005

[35] Martinez MJM, Pardo D, Velazquez JE. 2d bipolar Monte Carlo calculation of current fluctuations at the onset of quasisaturation of a Si BJT. Physica B: Condensed Matter. 1999;272:263-266. DOI: 10.1016/S0921-4526(99)00282-3

[36] Martin MJ, Pardo D, Velazquez JE. Microscopic analysis of the influence of ge profiles on the current-noise operation mode of n-si/p-si1-xgex heterostructures. Semiconductor Science and Technology. 2000;15:277-285. DOI: 10.1088/0268-1242/15/3/310

[37] Schaffler F. High-mobility si and ge structures. Semiconductor Science and Technology. 1997;12:1515-1549. DOI: 10.1088/0268-1242/12/12/001 
[38] Roldan JB, Gamiz F, Cartujo-Cassinello R, Cartujo P, Carceller JE, Roldan A. Strained-si on si1-xgex mosfet mobility model. IEEE Transactions on Electron Devices. 2003;50:14081411. DOI: 10.1109/TED.2003.81347

[39] Gaspari V, Fobelets K, Velazquez-Perez JE, Ferguson R, Michelakis K, Despotopoulos S, Papavassilliou C. Effect of temperature on the transfer characteristic of a $0.5 \mathrm{mu}$ m-gate Si:Sige depletion-mode n-modfet. Applied Surface Science. 2004;224:390-393. DOI: doi. org/10.1016/j.apsusc.2003.08.066

[40] Meziani YM, Garcia E, Calvo J, Diez E, Velázquez E, Fobelets K, Knap W. Detection of Terahertz Radiation from Submicron Plasma Waves Transistors. In: Unil Perera AG, editor. Book Bolometers. Rijeka, Croatia: Intech; 2012. pp. 137-150, ISBN 978-953-51-0235-9

[41] Lu JQ, Shur M. Terahertz detection by high-electron-mobility transistor: Enhancement by drain bias. Applied Physics Letters. 2001;78:2587-2588. DOI: 10.1063/1.1367289

[42] Popov VV, Fateev DV, Otsuji T, Meziani YM, Coquillat D, Knap W. Plasmonic terahertz detection by a double-grating-gate field-effect transistor structure with an asymmetric unit cell. Applied Physics Letters. 2011;99:243504. DOI: 10.1063/1.3670321

[43] Drexler C, Dyakonova N, Olbrich P, Karch J, Schafberger M, Karpierz K, Mityagin Y, Lifshits MB, Teppe F, Klimenko O, Meziani YM, Knap W, Ganichev SD. Helicity sensitive terahertz radiation detection by field effect transistors. Journal of Applied Physics. 2012;111:124504. DOI: 10.1063/1.4729043

[44] Sakowicz M, Lusakowski J, Karpierz K, Grynberg M, Gwarek W, Boubanga S, Coquillat D, Knap W, Shchepetov A, Bollaert S. A high mobility field-effect transistor as an antenna for sub-THz radiation. AIP Conf. Proc. 2010;1199:503. DOI: 10.1063/1.3295528

[45] Veksler DB, Muravjov AV, Kachorovskii VY, Elkhatib TA, Salama KN, Zhang XC, Shur MS. Imaging of field-effect transistors by focused terahertz radiation. Solid-State Electronics. 2009;53:571. DOI: 10.1016/j.sse.2009.04.004

[46] Sakowicz M, Lusakowski J, Karpierz K, Grynberg M, Gwarek W, Boubanga S, Coquillat D, Knap W, Shchepetov A, Bollaert S. A high mobility field-effect transistor as an antenna for sub-THz radiation. AIP Conf. Proc. 2010;1199:503-504. DOI: 10.1063/1.3295528

[47] Rogalsky A, Sizov F. Terahertz detectors and focal plane arrays. Opto-Electronics Review. 2011;19(3):346-404. DOI: 10.2478/s11772-011-0033-3 
